\title{
Dimensión social de la práctica docente. Estudio comparado entre la Universidad de Cienfuegos (Cuba) y la Universidad Autónoma del Estado de Hidalgo (México)
}

\author{
George Reyes, Carlos Enrique \\ Universidad Autónoma del Estado de Hidalgo, México \\ cgeorgemx@gmail.com
}

Resumen - Las Tecnologías de la Información y la Comunicación (TIC) son herramientas que paulatinamente se han integrado en las actividades pedagógicas que realizan los docentes con la premisa de que producirán cambios en la forma en la que se enseñan los contenidos curriculares, sin embargo, suponer que en todas las instituciones educativas existe una suficiencia de infraestructura tecnológica para llevar a cabo esta actividad, o bien que se utilizarán con eficiencia los dispositivos que se tienen al alcance, es arriesgado. Con el propósito de valorar la forma en la que se han integrado las TIC en la práctica docente George y Veytia (2017) diseñaron el modelo ITIC-PD (Integración de las TIC en la Práctica Docente) que permite identificar desde diversas dimensiones los niveles en los que se incorporan las tecnologías en la labor educativa. El objetivo de esta investigación fue comparar desde la Dimensión Social de este modelo el acercamiento que tienen los docentes a la infraestructura TIC y si dichos acercamientos están produciendo cambios pedagógicos, para lograrlo se utilizó un cuestionario y se aplicaron entrevistas a docentes de dos universidades latinoamericanas. Los hallazgos mostraron que aun cuando existen diferencias notables respecto a la infraestructura tecnológica con la cuentan ambas instituciones, estas no generan cambios pedagógicos trascendentales, por lo que se concluye que presuponer que la presencia de las tecnologías conlleva por sí misma una transformación educativa es una falacia.

Palabras clave: Estudio comparado; TIC; práctica docente; Cuba; México;
Abstract - Information and Commu- nication Technologies (ICT) are tools that have been gradually inte- grated into the pedagogical activi- ties carried out by teachers with the premise that they will produce changes in the way curricular con- tents are taught, however, suppose that In all educational institutions there is a sufficient technological infrastructure to carry out this acti-

Interconectando Saberes, 2019

ISSN: 2448-8704
Fecha de Recepción: 30 de marzo de 2019

Fecha de Aceptación: 21 de junio de 2019

Fecha de Publicación: 30 de junio de 2019 
vity, or that devices that are available are used efficiently, it is risky. With the purpose of assessing the way in which ICTs have been integrated into teaching practice, George and Veytia (2017) designed the ITIC-PD model (Integration of ICT in Teaching Practice) that allows identifying the levels in different dimensions those that incorporate technologies in educational work. The objective of this research was to compare the approach of teachers to ICT infrastructure from the Social Dimension of this model and if these approaches are producing pedagogical changes, a questionnaire was used and interviews were conducted with teachers from two Latin American universities. The findings showed that even though there are notable differences regarding the technological infrastructure with both institutions, they do not generate transcendental pedagogical changes, so it is concluded that presupposing that the presence of technologies entails an educational transformation by itself is a fallacy.

Keywords: Comparative study; ICT; teaching practice; Cuba; Mexico;

\section{INTRODUCCIÓN}

La integración de las Tecnologías de la Información y la Comunicación (TIC) desde hace ya muchos años ocupan un lugar fundamental en el escenario educativo, de esta forma los docentes cotidianamente acuden a los dispositivos digitales que tienen a su alcance para reali- zar sus actividades profesionales, así, pueden acceder a volúmenes extensos de información o bien a herramientas que agilizan el proceso de enseñanza, en especial en la educación superior se sostiene que pueden aplicarse para elaborar materiales didácticos, propiciar la comunicación entre alumnos y docentes, generar nuevas estrategias pedagógicas, entre otras actividades (UNESCO, 2013).

Evaluar la forma en la que se han integrado estas herramientas en la educación ha sido objeto de estudio en años recientes en distintas investigaciones (Pérez y Rodríguez, 2016, Romero, et. al. 2017, Martínez, Hinojo y Rodriguez, 2017, Blanco, 2018), que desde diversas aristas han intentado dilucidar qué implica el uso de dispositivos digitales en la generación de nuevas pedagogías.

Sin embargo, hasta el momento no se sabe con precisión si la suficiencia en infraestructura tecnológica en las universidades o bien la que posee el propio docente es suficiente para desarrollar nuevos diseños pedagógicos, en este 
sentido, George y Veytia (2017) realizaron una propuesta para evaluar el impacto de las TIC (ITIC-PD) que se distingue por hacer una imbricación entre el uso de las TIC en la educación y las dimensiones de la práctica docente, en particular la dimensión social valora el acceso que se tiene a herramientas digitales y la percepción de la eficiencia pedagógica que se logra cuando se hace uso de ellas.

Esta investigación aborda mediante la metodología de los estudios comparados la aplicación de la propuesta mencionada en dos universidades públicas latinoamericanas desde el enfoque del acceso a la infraestructura y de las percepciones de los docentes respecto al uso pedagógico que les asignan a las tecnologías que tienen a su alcance con el fin de identificar si están obteniendo cambios en la enseñanza.

El artículo se conforma de un primer apartado donde se describen las particularidades del modelo ITIC-PD con el fin de contextualizar al lector sobre los niveles que lo caracterizan y los descriptores que lo componen, en el segundo se enuncian las precisiones metodologías que delimitaron el estudio comparado, posteriormente se realiza la aplicación y el análisis de la información obtenida en la comparación y se redactan a modo de resultados los cambios pedagógicos identificados, se finaliza con las conclusiones.

\section{LA DIMENSIÓN SOCIAL DESDE EL MODELO ITIC-PD}

Analizar como se han incorporado las TIC en la educación requiere de un marco de referencia con el que se pueda identificar, primero, si el docente tiene a su alcance la infraestructura tecnológica necesaria para hacer operativos sus propósitos pedagógicos mediados por el uso de dispositivos digitales, y segundo, examinar que cambios en la forma de enseñar se están logrando al usar herramientas digitales en las actividades sustantivas del profesor.

Con el fin de contribuir para dilucidar los puntos anteriores, George y Veytia (2017) diseñaron un modelo con el que a través de la imbri- 
cación de los niveles competenciales del uso de las TIC generalmente aceptados por la comunidad científica (adopción y transformación), las etapas de avance (uso, asimilación, reorientación y evolución) y las dimensiones de la práctica docente (social, institucional, personal, valoral, interpersonal y didáctica) se pueden identificar cambios en la enseñanza.

El modelo llamado ITIC-PD (ver Figura 1) identifica como principal actor al docente, así, la dimensión institucional es el componente contextual que delimita su actividad a los marcos normativos de la institución educativa, la dimensión social, hace referencia al espacio de incidencia más inmediato de la labor docente relacionado con la infraestructura tecnológica a la que tiene acceso, mientras que la personal e interpersonal se vinculan con la trayectoria individual y profesional así como las relaciones que ha construido con sus semejantes, por otra parte, la dimensión didáctica y valoral se centra en las decisiones pedagógicas que toma respecto a incorporar las TIC y a la afinidad que tiene con éstas herramientas.

\section{Figura 1. Modelo ITIC-PD}

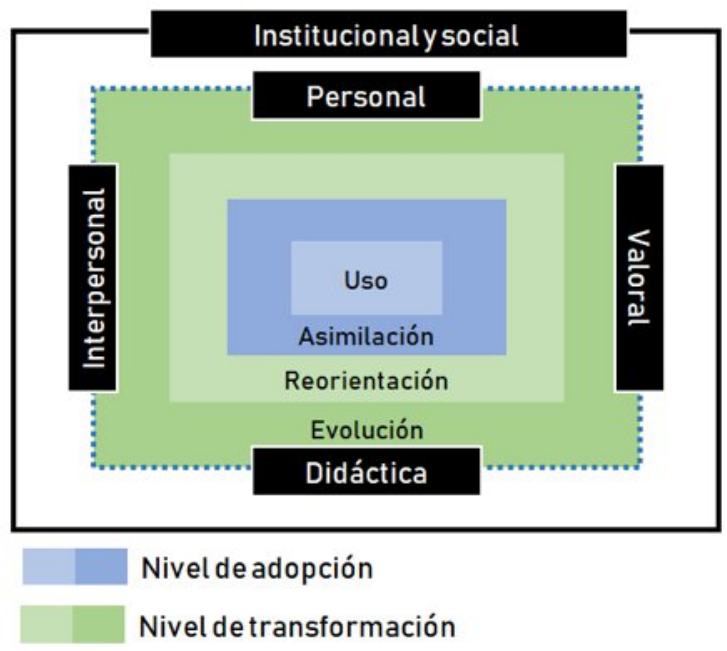

Fuente: George y Veytia (2017).

Para la elaboración de este estudio, se consideró analizar la dimensión social, ya que de acuerdo con Alfaro, Fernández y Alvarado (2014) la práctica docente surge y se desarrolla en contextos sociales complejos, donde la tecnología ha adquirido un papel relevante en la educación (Cela et. al., 2017), sin embargo, esta incorporación no es permanente, sino que se encuentra en constante construcción debido a las presiones que reciben de los estudiantes y la propia institución educativa en el sentido de utilizar la infraestructura tecnológica que se encuentra disponible en el recinto escolar. 
De esta forma la práctica docente se encuentra influida por el contexto socioeducativo donde se desarrolla la docencia (Díaz y Hernández, 2010) y está fuertemente influida por variables como el entorno económico y para el caso de esta investigación por la infraestructura tecnológica a la que tiene acceso el docente, ya sea en el plano institucional o personal, los descriptores de esta dimensión se muestran en la Tabla 1.
En este sentido, la investigación aborda las percepciones de los docentes respecto a la capacidad de la infraestructura instalada en la universidad para llevar a cabo actividades de enseñanza mediadas por la tecnología, también indaga acerca de sus posibilidades de acceso para aprovechar las herramientas que se encuentran en el recinto escolar y finalmente identifica la forma en que utiliza (o no utiliza) las TIC para cambiar sus prácticas escolares.

\section{Tabla 1 Descriptores de incorporación de las TIC. Dimensión social.}

\begin{tabular}{|c|c|c|c|}
\hline \multicolumn{2}{|c|}{ Nivel de adopción } & \multicolumn{2}{|c|}{ Nivel de transformación } \\
\hline $\begin{array}{c}\text { Etapa del nivel: } \\
\text { Aproximación }\end{array}$ & $\begin{array}{l}\text { Etapa del nivel: } \\
\text { Asimilación }\end{array}$ & $\begin{array}{l}\text { Etapa del nivel: } \\
\text { Reorientación }\end{array}$ & $\begin{array}{c}\text { Etapa del nivel: } \\
\text { Evolución }\end{array}$ \\
\hline $\begin{array}{l}\text { a) Identifica que la } \\
\text { institución escolar } \\
\text { no tiene } \\
\text { infraestructura } \\
\text { tecnológica para } \\
\text { diseñar escenarios } \\
\text { de aprendizaje } \\
\text { relacionados con } \\
\text { el uso de las TIC. } \\
\text { b) Identifica que } \\
\text { no posee } \\
\text { dispositivos } \\
\text { digitales ni } \\
\text { conectividad a } \\
\text { internet para } \\
\text { diseñar escenarios } \\
\text { de enseñanza } \\
\text { relacionados con } \\
\text { el uso de las TIC. } \\
\text { c) Se rehúsa a } \\
\text { utilizar las TIC en } \\
\text { la enseñanza. }\end{array}$ & $\begin{array}{l}\text { a) Reconoce que } \\
\text { la infraestructura } \\
\text { tecnológica en la } \\
\text { institución escolar } \\
\text { es escasa, lo que } \\
\text { no le permite } \\
\text { diseñar escenarios } \\
\text { de aprendizaje con } \\
\text { el uso de las TIC. } \\
\text { b) Reconoce que } \\
\text { su acceso } \\
\text { personal a } \\
\text { dispositivos } \\
\text { digitales y } \\
\text { conectividad es } \\
\text { escaso por lo que } \\
\text { no puede diseñar } \\
\text { escenarios de } \\
\text { enseñanza con el } \\
\text { uso de las TIC. } \\
\text { c) Utiliza las TIC } \\
\text { de forma } \\
\text { intermitente y sin } \\
\text { fines pedagógicos. }\end{array}$ & $\begin{array}{l}\text { a) Organiza sus } \\
\text { posibilidades de } \\
\text { acceder a la } \\
\text { infraestructura } \\
\text { tecnológica en la } \\
\text { institución escolar } \\
\text { para implementar } \\
\text { escenarios de } \\
\text { enseñanza con el } \\
\text { uso de las TIC. } \\
\text { b) Organiza sus } \\
\text { posibilidades para } \\
\text { acceder a } \\
\text { dispositivos } \\
\text { digitales y } \\
\text { conectividad para } \\
\text { implementar } \\
\text { escenarios de } \\
\text { enseñanza con el } \\
\text { uso de las TIC. } \\
\text { c) Utiliza las Tic } \\
\text { de forma básica y } \\
\text { les asigna una } \\
\text { función } \\
\text { pedagógica. }\end{array}$ & $\begin{array}{l}\text { a) Evalúa su } \\
\text { práctica docente a } \\
\text { partir de la } \\
\text { implementación de } \\
\text { escenarios de } \\
\text { enseñanza } \\
\text { mediados por la } \\
\text { infraestructura } \\
\text { tecnológica presente } \\
\text { en la institución } \\
\text { escolar. } \\
\text { b) Evalúa su } \\
\text { práctica docente a } \\
\text { partir de la } \\
\text { implementación de } \\
\text { escenarios de } \\
\text { enseñanza usando } \\
\text { su acceso a } \\
\text { dispositivos digitales } \\
\text { y conectividad. } \\
\text { c) Utiliza las Tic de } \\
\text { forma eficiente para } \\
\text { desarrollar } \\
\text { actividades } \\
\text { pedagógicas. }\end{array}$ \\
\hline
\end{tabular}




\section{PreCisiones METODOLÓGICAS}

De acuerdo con Schriewer (1993), la comparación es un proceso típico y esencialmente humano que representa una acción constante en cualquier actividad, es decir, se comparan precios, servicios, países $y$ todo tipo de hechos que suceden en la vida, en el sentido científico la comparación se sustenta en un pensamiento relacional donde se pueden hacer comparaciones simples y complejas, en los siguientes apartados se delimita el alcance y las etapas del estudio así como los criterios de selección de los participantes.

Determinación del alcance del estudio comparado

Bray, Adamson y Mason (2010) recuperan distintos enfoques y métodos para hacer estudios comparados que van desde la comparación de espacios escolares hasta la comparación de innovaciones educativas, los autores sugieren como marco de referencia para el análisis de estudios comparados en educación el cubo de Bray y Thomas (1995), con el fin de hacer operable el cubo (ver Figura 2) se delimitó que para este estudio se seleccionaría el nivel 2 de localización geográfica que corresponde la comparación entre países (México y Cuba), respecto a los grupos demográficos no locativos fue seleccionado otros grupos, ya que los sujetos de comparación son docentes pertenecientes a dos universidades públicas estatales, la Universidad Autónoma del Estado de Hidalgo (UAEH, México) y la Universidad de Cienfuegos (UCF, Cuba), mientras que en los aspectos de la educación y la sociedad se eligió enseñanza/métodos.

Figura 2. Operacionalización del cubo de Bray y Thomas (1995) para la investigación.

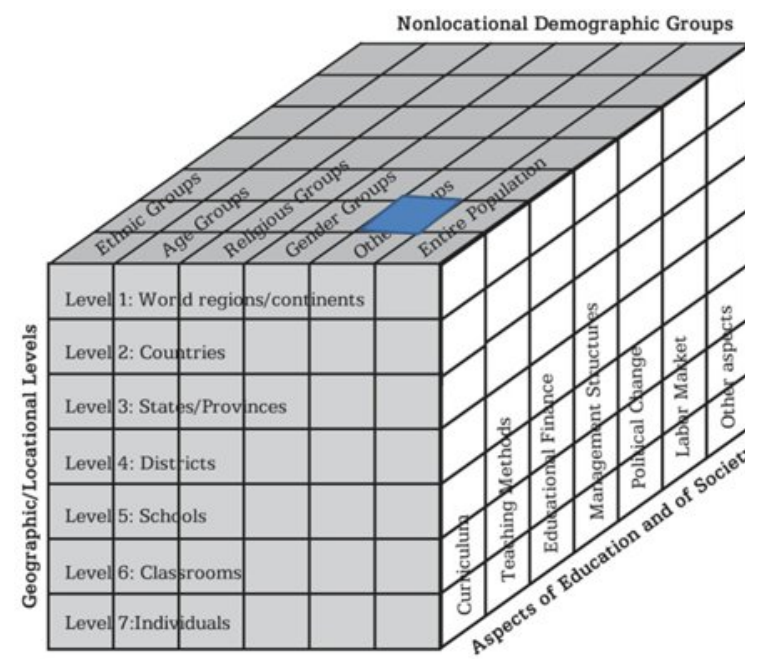

Fuente: Elaboración propia a partir de Bray y Thomas (1995). 
Tabla 2. Etapas llevadas a cabo en el estudio comparado.

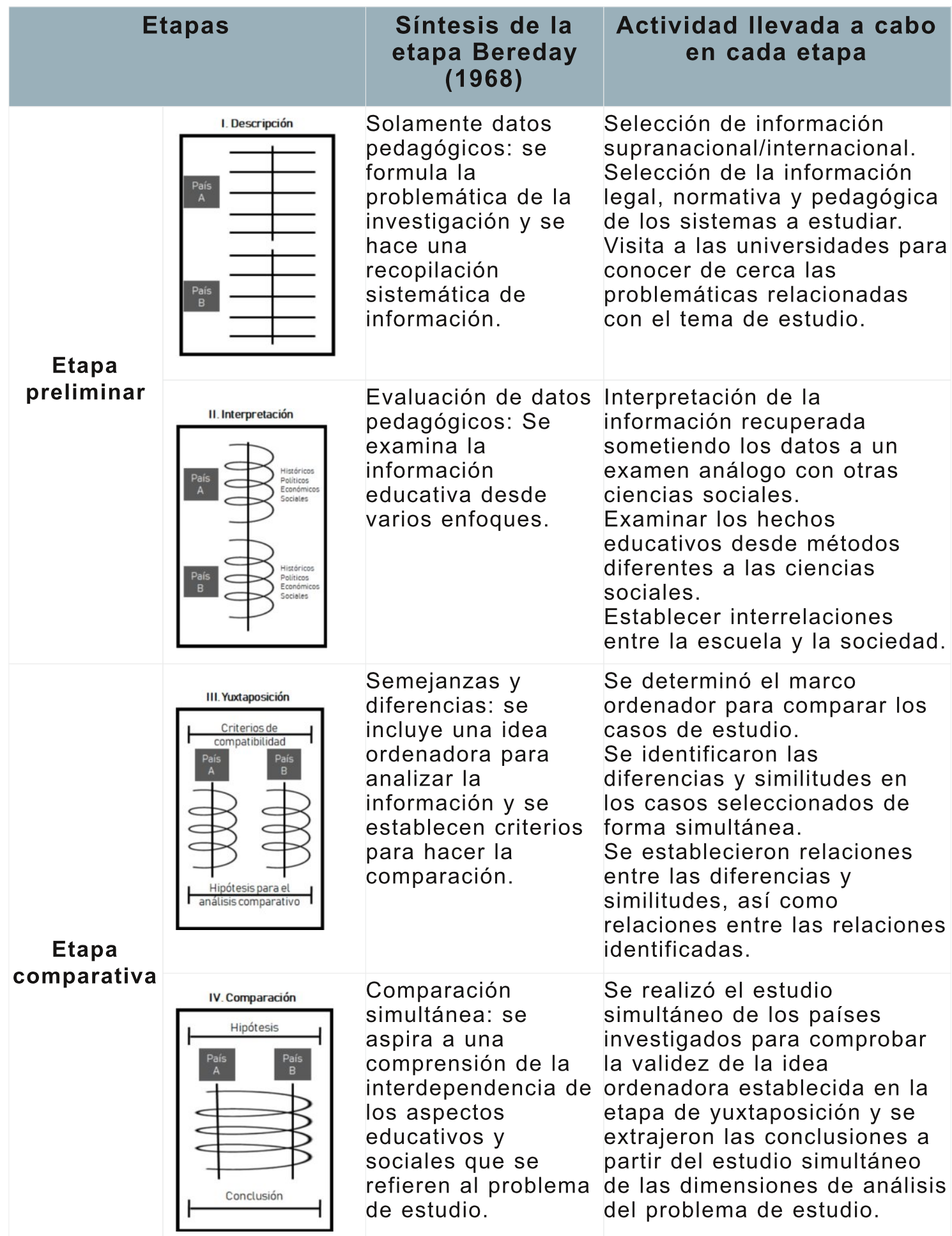

Fuente: Elaboración propia a partir de Hilker (1964) y Bereday (1983). 
Etapas del estudio comparado

En cuanto a los momentos por los que se debe transitar para realizar un estudio comparado, Hilker (1964), Bereday (1968) y Raventós (1983) coinciden al señalar que la comparación debe llevarse a cabo en 4 etapas de investigación: 1) descripción, 2) interpretación, 3) Yuxtaposición y 4) comparación, en Tabla 2 se muestran las actividades realizadas para afrontar en este estudio cada una de estas etapas:

Selección de participantes para el estudio comparado

Los participantes del estudio fueron docentes de tiempo completo de la Universidad de Cienfuegos (Cuba) y de la Universidad Autónoma del Estado de Hidalgo (México), se eligieron a partir de un muestro no probabilístico por conveniencia (Creswell y Plano, 2011), considerando como criterio de selección la facilidad operativa por la que se pudieron aplicar encuestas y entrevistas a los profesores.

La muestra quedo conformada por docentes de la Universidad de Cienfuegos adscritos al Centro de Estudios de la Didáctica y Dirección de la Educación Superior (CEDDES), en la Universidad autónoma del Estado de Hidalgo aceptaron participar docentes adscritos al Centro de Investigación en Ciencias y Desarrollo de la Educación (CINCIDE), en la Tabla 3 se muestra el porcentaje de participación.

Selección de técnicas de investigación

Para realizar estudios comparados Stake (1998) recomienda hacer uso de técnicas cualitativas, mientras que Landman (2011) propone el uso de técnicas cuantitativas para aquellos que consideren a muchos

Tabla 3. Participantes en el estudio comparado.

\begin{tabular}{|c|c|c|c|}
\hline Escenario & $\begin{array}{c}\text { Total, de } \\
\text { docentes } \\
\text { adscritos }\end{array}$ & $\begin{array}{c}\text { Total, de } \\
\text { docentes que } \\
\text { participaron }\end{array}$ & Porcentaje \\
\hline CEDDES & 14 & 11 & $85.71 \%$ \\
\hline CINCIDE & 21 & 16 & $76.19 \%$ \\
\hline
\end{tabular}

Fuente: Elaboración propia. 
países y cualitativas para pocos países, sin embargo, Bourdieu (2012) menciona que los investigadores no deben someterse al monoteísmo metodológico, sino que deben utilizar los instrumentos que les sean útiles para conocer la realidad que están buscando, de la misma forma, Cook y Reichardt (1986), afirman que:

[...] un investigador no tiene por qué adherirse ciegamente a uno de los paradigmas de polos opuestos que han sido etiquetados como "cualitativos" y "cuantitativos", sino que puede elegir libremente una mezcla de atributos de ambos paradigmas de manera que se adapten mejor a las demandas del problema de investigación en curso ( $p$. 43).

Por ello, fue utilizado en primer término un cuestionario de escala tipo Likert que permite conocer el grado de conformidad de un encuestado con las afirmaciones propuestas por el investigador, el objetivo fue recuperar una imagen estadística de el asentimiento de los docentes respecto a su acercamiento con la infraestructura TIC. Los resultados obtenidos sirvieron como criterio para aplicar una entrevista semi-estructurada para profundizar las opiniones recabadas. La utilización de esta estrategia se fundamenta con la idea multimetodológica de la investigación cualitativa (Denzin y Lincoln, 2012), ya que al usar técnicas estadísticas se pueden obtener distribuciones de una población entre categorías, que pueden ser empleadas en descripciones o verificaciones de la realidad (Glaser y Strauss, 1967).

\section{Análisis de los datos}

Se utilizó el software SPSS versión 22.0 para analizar estadísticamente los datos recuperados normalizando los ítems en una escala de 1 a 4 , donde el valor 1 representa una menor incorporación de las TIC y 4 una mayor incorporación, para observar las diferencias se compararon los resultados con los descriptores del modelo ITIC-PD para cada universidad y se identificaron las diferencias significativas mediante un análisis radial (AR), ya que éste se utiliza principalmente para hacer la comparación de datos (Vargas, 1995). 
Posteriormente se realizó el análisis cualitativo usando el software Atlas. Ti versión 6, siguiendo como estrategia metodológica la identificación de patrones y relaciones de datos (Strauss y Corbin, 2002) a partir del análisis del discurso de los docentes. Es decir, se realizó un proceso de codificación que consiste en comparar la información recolectada mediante la asignación de categorías el cual consta de 4 etapas que no necesariamente son secuenciales: 1) la codificación abierta, 2) la codificación axial, 3) la codificación selectiva y 4 ) la estructuración de los hallazgos.

\section{RESUlTADOS}

La presentación de los resultados se lleva a cabo de acuerdo con las etapas de los estudios comparados, cabe aclarar que la etapa de descripción e interpretación se desarrolló de forma preliminar mediante la búsqueda y análisis de la información contextual de las universidades y docentes seleccionados, por lo que en este articulo se parte de la etapa de Yuxtaposición y continúa con la comparación.
Yuxtaposición de la dimensión social del modelo ITIC-PD

De acuerdo con el modelo ITIC-PD, en la dimensión social la UCF se encuentra en una etapa de asimilación y un nivel de adopción con 1.88 puntos mientras que la $U A E H$ se encuentra en la etapa de reorientación y nivel de transformación con 2.56, muy cercano a la etapa de evolución, en la Tabla 4 se muestran los porcentajes alcanzados en cada universidad.

Los puntajes significan que en la UAEH: 1) el docente considera que la infraestructura tecnológica es suficiente para generar estrategias de uso de las TIC en la docencia, 2) que tiene suficientes dispositivos digitales y conectividad para generar estrategias pedagógicas, y 3 ) que usa de forma eficiente las TIC que tiene a su disposición.

Mientras que, en la UCF, el docente considera que: 1) la infraestructura tecnológica en la universidad es escasa para generar estrategias pedagógicas, 2) tiene escasos dispositivos digitales y conectividad para generar estrategias 
Tabla 4. Niveles y etapas de incorporación de las TIC en la Dimensión Social.

\begin{tabular}{|c|c|c|c|c|c|}
\hline Código & Indicador & $\begin{array}{l}\text { Puntaje } \\
\text { UCF }\end{array}$ & $\begin{array}{l}\text { Porcentaje } \\
\text { UCF }\end{array}$ & $\begin{array}{l}\text { Puntaje } \\
\text { UAEH }\end{array}$ & $\begin{array}{l}\text { Porcentaje } \\
\text { UAEH }\end{array}$ \\
\hline E11 & $\begin{array}{l}\text { Infraestructura TIC de } \\
\text { la universidad }\end{array}$ & 1.55 & $39 \%$ & 2.13 & $53 \%$ \\
\hline E21 & $\begin{array}{l}\text { Infraestructura TIC del } \\
\text { docente }\end{array}$ & 1.73 & $43 \%$ & 2.38 & $59 \%$ \\
\hline E31 & $\begin{array}{l}\text { Uso eficiente de las } \\
\text { TIC en la docencia }\end{array}$ & 2.45 & $61 \%$ & 3.19 & $80 \%$ \\
\hline \multicolumn{2}{|c|}{$\begin{array}{l}\text { Promedio de la Dimensión } \\
\text { Social }\end{array}$} & 1.88 & $47.73 \%$ & 2.56 & $74.78 \%$ \\
\hline \multicolumn{2}{|c|}{$\begin{array}{l}\text { Nivel de Incorporación de las } \\
\text { TIC en la Dimensión Social }\end{array}$} & \multicolumn{2}{|c|}{ Adopción } & \multicolumn{2}{|c|}{ Transformación } \\
\hline \multicolumn{2}{|c|}{ Cambios en la práctica docente } & \multicolumn{2}{|c|}{ Cambios simulados } & \multicolumn{2}{|c|}{ Cambios emergentes } \\
\hline
\end{tabular}

\section{Fuente: Elaboración propia.}

de uso de las TIC en la docencia, y 3) usa de forma suficiente las TIC que tiene a su disposición.

Lo anterior, de acuerdo con el modelo ITIC-PD ubica a los docentes de la UAEH en nivel de transformación de la práctica docente con el uso de las tecnologías que generan cambios emergentes en la enseñanza, mientras que en la UCD se alcanza el nivel de adopción con una mayoría de cambios simulados que se basan en la sustitución de herramientas (proyector por pizarrón, material impreso por material digital, etc.) En la Gráfica 1 se representa la dispersión de las opi- niones de los encuestados en ambas universidades.

En esta dispersión se observa qué los docentes $1,5,7,9$ y 11 de la UCF tienen una mayor tendencia para realizar actividades con infraestructura tecnológica que tienen disponible al igual que los docentes $5,7,11,13$ y 15 de la UAEH se encuentran en este supuesto, sin embargo, al realizar el análisis de las entrevistas las narraciones de los docentes contradicen las percepciones obtenidas en la encuesta, como evidencia de esta afirmación se generó el esquema representacional de la Figura 3. 


\section{Gráfica 1. Representación del modelo ITIC-PD en la Dimensión Social.}

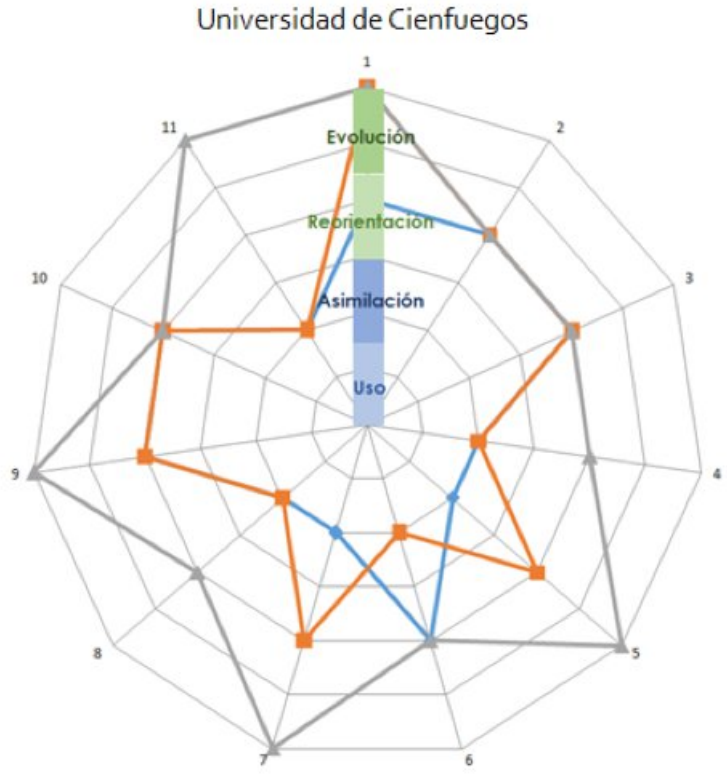

—E11) Considera que la universidad tiene los dispositivos y la conexión a internet que usted necesita para utilizar las TIC en sus actividades docentes.

-E21) Tiene usted los dispositivos y la conexión a Internet que necesita para utilizar las TIC en su práctica docente.
Universidad Autónoma del Estado de Hidalgo

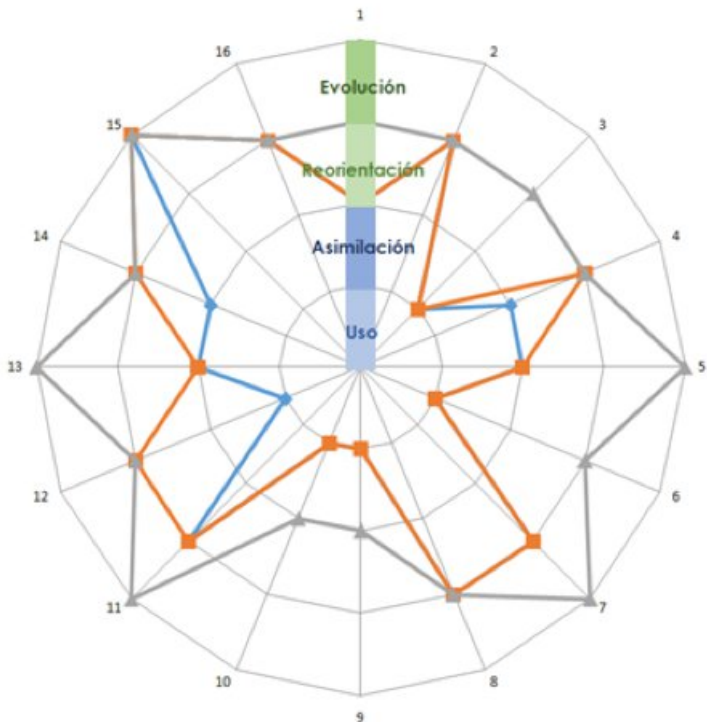

-E31) Se identifica como un profesor que desarrolla su actividad docente utilizando de forma eficiente las TIC como parte de sus estrategias de enseñanza.

Fuente: Elaboración propia.

Figura 4. Esquema representaciones de las narraciones de los docentes.

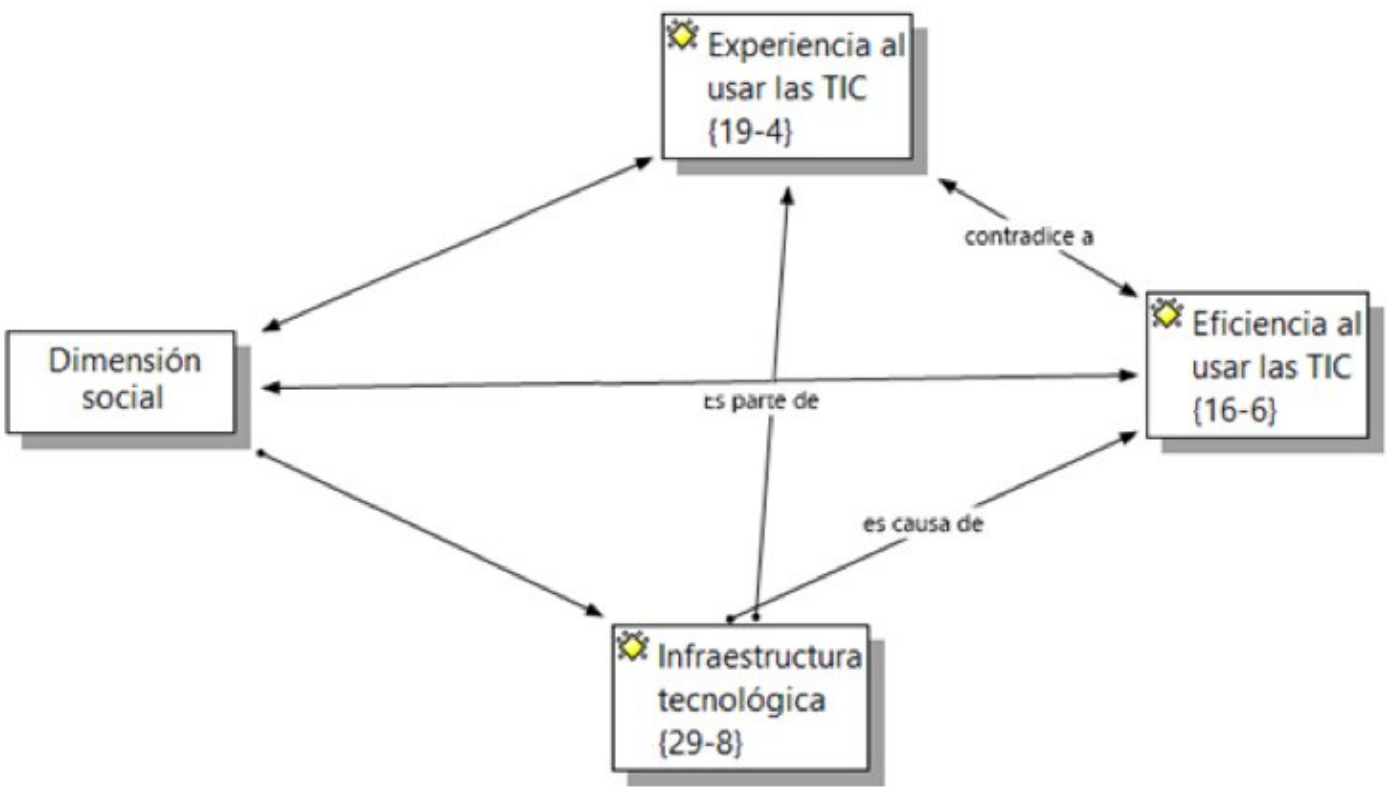

Fuente: Elaboración propia a partir del esquema representacional de la codificación teórica de la Dimensión Social. 
Las representaciones de las narraciones de los docentes pueden categorizarse como: 1) la infraestructura tecnológica como elemento imprescindible para cambiar la práctica académica es causa de que existan opiniones diferentes sobre 2) la eficiencia para usar las TIC que tiene a su disposición el docente, y que es parte de 3) las experiencias de los docentes al usar herramientas digitales, que en varios casos son contradictorias como se argumentará en los sucesivos párrafos:

\section{1) Infraestructura tecnológica}

Ramírez y Casillas (2015) mencionan que la adquisición de equipo de cómputo, proyectores y otros dispositivos depende de la percepción de las necesidades de encargados de programas de equipamiento y de la autorización del área de finanzas de las universidades, lo que hace que la vida útil de los aparatos tecnológicos se reduzca o que estos no sean consistentes con las necesidades de los docentes, por ello, es importante indagar cómo los entrevistados valoran el estado actual de la infraestructura física y del equipamiento tecnológico en la universidad, así como del uso que hacen de ellos (Lizarazo y Andión, 2013), las narraciones fueron homogéneas en el sentido de calificar insuficiente el acceso a dispositivos digitales:

UAEH D7: [...] se han hechos grandes esfuerzos, pero yo creo que no es suficiente, hay que seguir invirtiendo en alguna parte de la infraestructura, y poco a poco ir subsanando lo que nos falta, por ejemplo, los laboratorios de cómputo nos ayudan para que los alumnos realicen algunas actividades utilizando las TIC, pero muchas veces tampoco son suficientes.

UCFD9: [...] en algunos momentos la infraestructura suficiente, en otros no, pues nosotros estamos muy limitados con la capacidad de la Internet, no es un problema de la universidad es un problema del pais por todo lo que tú conoces del bloqueo, esto es un problema serio, pero el docente sabe adecuarse a las condiciones que prevalecen en el pais.

Los docentes de ambas universidades perciben que se han realizado esfuerzos institucionales para disponer de herramientas tecnológi- 
cas, sin embargo, los consideran limitados ya que el acceso a dispositivos digitales se encuentra, en el caso de la UAEH en los laboratorios de cómputo, mientras que en la UCF se aprecia una mayor problemática para tener un acercamiento con la tecnología por la condición socioeconómica derivada del bloqueo comercial de los Estados Unidos de Norteamérica a $\mathrm{Cu}$ ba.

$\mathrm{Si}$ bien, las universidades se sitúan en contextos de desarrollo económico diferentes, en sus legislaciones está presente la importancia del uso de las tecnologías como elemento de transformación y mejoramiento de la sociedad (Cárdenas, et.al., 2017), en el caso de la UAEH existe la percepción de que los cambios en la enseñanza que se planifican no pueden llevarse a cabo en las aulas, mientras que en la UCF se considera como una inconsistencia la relación entre los mandados del Ministerio de Educación y la realidad para usar TIC.

UAEHD 11: [...] no, siento de entrada y de manera general que la universidad no tiene la infraestructura suficiente para que los docentes utilicemos las TIC, tenemos problemas del impacto de la actividad docente se da en las aulas, no sirve de mucho que en el cubiculo planifiques y desarrolles una actividad donde los alumnos tengan que utilizar la tecnología para desarrollar conocimientos y cuando bajas al aula, pues ya no hay Internet.

UCFD1: [...] ha mejorado la infraestructura, pero poco, existe un gran desvario entre lo que tú tienes como docente, lo que te solicita el Programa de Informatización de la Sociedad Cubana, lo que te pide el Ministerio de Educación, así no puedes alterar el sistema de clases, tienes que seguir con la clase tradicional.

Lo anterior afirma que las acciones relativas para disponer de una infraestructura suficiente han sido azarosas, en parte porque son las mismas universidades las que bajo sus propios criterios de crecimiento tecnológico se han encargado su desarrollo (Casillas, Ramírez, Carvajal y Valencia, 2016), y no han considerado las necesidades básicas ni las disciplinares de los docentes, de esta forma para que la presencia de las tecnologías genere cambios es necesario seguir apostando por incrementar la dotación 
tecnológica no solamente en las áreas administrativas, sino también en las aulas y espacios de convivencia entre docentes y estudiantes.

\section{2) Eficiencia al usar las TIC}

En relación con el uso de las TIC, la eficiencia es el momento en el que se hace un uso correcto de los recursos asignados, no solo a nivel financiero, sino en el uso de las tecnologías para producir cambios en la enseñanza y con ello mejorar la calidad de los logros educativos (OEI, 2018), en las narraciones la perspectiva de los docenes es que no se está logrando esta eficiencia. El ejemplo más significativo se encuentra en las aulas de la UAEH, ya que dispositivos digitales como el pizarrón electrónico han sido aprovechados durante un corto periodo de tiempo y después olvidados.

UAEHD9: [...] era muy dinámico [usar los pizarrones electrónicos] porque te daban unas estuches con los controles y con los plumones para que pudieras trabajar con ellos, pero se vinieron para abajo, quedaron abandonados, ese fue dinero que se fue la basura porque ahora ya nadie los utiliza, porque te deben de instalar el software en tu computadora y hay maestros que no llevan computadora al aula de clases, hay maestros que aun cuando llevan su computadora pues no están interesados en utilizar el pizarrón electrónico porque no saben usarlo, además que la capacitación fue muy rápida, sólo dijeron se enciende si se conecta el equipo y puedes hacer esto y nada más.

UAEHD11: [...] ahí está el pizarrón [electrónico], el docente no lo considera para impartir su cátedra por qué pues no sabe utilizarlo, no se le sabe sacar provecho, se le pierde el sentido de utilizarlo porque no hay nadie que te capacite para hacerlo.

Por lo que adquirir dispositivos digitales en las universidades y capacitar a los docentes para usarlos no significa que se esté estableciendo un vínculo entre la tecnología y las estrategias de enseñanza, de igual forma, es inconsistente que el docente utilice un pizarrón electrónico, cuando no cuenta con un el software ni un equipo personal para operarlo, así como tampoco soporte técnico: 
UAEHD5: [...] a nadie jamás he visto utilizar un pizarrón digital aqui, son pizarrones que requieren la plumita especial, que nadie sabe dónde quedaron, es un equipo que está detenido, ¿sabes por qué?, pues porque también necesitas tener una lap para conectarlo y no todos los docentes tienen o quieren traer sus computadoras para hacerlo, y aún si la trajeras necesitas el programa para operarlo y nadie sabe dónde está ese programa.

En la UCF también se observa que la eficiencia para usar las tecnologías no está relacionada con la ausencia de infraestructura tecnológica, sino con una apreciación de escasa alfabetización digital para operar dispositivos digitales que ya se encuentran en las aulas, por lo que existe un desfase entre el uso de los dispositivos y los esquemas de enseñanza que regularan las prácticas docentes:

UCFD1: [...] si las usáramos como tendría que ser, serian insuficientes, en la universidad yo creo que los que no estamos ocupando las TIC adecuadamente somos nosotros, por qué no tenemos los conocimientos que nos permitan saber utilizar lo que tenemos.
UCFD9: [...] el problema no es si hay TIC o no hay TIC, el problema es que la mayoría de las veces no se usa de forma eficiente, muchos docentes no están alfabetizados digitalmente, lo que digo es que, si mañana tenemos una lap por docente o una Tablet las clases van a seguir igual, se necesita más que capacitación una alfabetización.

Lo anterior demuestra que no se están generando cambios en la práctica docente ya que no existe una percepción de capacidad o aptitud para incorporar las TIC con eficiencia en el escenario educativo, lo anterior resulta preocupante ya que puede originarse una marginación tecnológica dando por resultado menos posibilidades para desarrollarse y desenvolverse en todos los niveles sociales (Cabero, 2014).

3) Experiencia con el uso de las TIC

En estrecha vinculación con lo anterior, los docentes mencionan que sus experiencias profesionales con el uso de las tecnologías no han sido del todo favorables, en este sentido, en la UAEH prevalece la 
idea de que no se logra cambiar la enseñanza debido a que no se está asumiendo el reto de crear diferentes estrategias pedagógicas con las TIC que se tienen en la institución:

UAEHD 11: [...] yo creo que estamos al revés, creo que deberíamos de explotar al máximo la infraestructura que tenemos disponible para que en ese sentido tuviéramos bases para exigir más y no decir: no incorporo las tic porque a veces Internet me falla, sino más bien tener estrategias donde quede muy declarado que vamos utilizar Internet, de qué forma lo vamos utilizar para qué lo vamos a utilizar, y qué beneficios va dar al estudiante para tener mejores aprendizajes.

Para que la incorporación se pueda dar de forma armónica debe prevalecer la conciencia de que primero deben consolidarse estrategias pedagógicas para utilizar las tecnologías disponibles, es decir, existir un conocimiento del uso educativo de estas herramientas, en la UCF esto es una tarea pendiente como se puede leer en el siguiente testimonio:
UCFD5: [...] mira, todo parte de la motivación y la planeación que hagas para llevar las TIC a tu aula, debe ser una planeación real, no puedes planear usar el internet en clase porque sabes que no lo hay, tampoco puedes pedir que todos los alumnos usen sus laps porque sabes que no las tienen, pero lo que si puedes hacer es tú como docente usar tu lap y el proyector para enriquecer tu enseñanza, crear tus estrategias, no pedir al estudiante que él sea el que determine como se va a generar la estrategia de enseñanza, es el estudiante.

De lo anterior se desprende que no solo es necesario disponer de dispositivos electrónicos, sino también debe existir un interés por hacer una planeación realista a partir de la infraestructura con la que se cuenta para que gradualmente se puedan aprovechar desde un enfoque pedagógico, de esta forma se observa que en ambas universidades la incorporación está fundamentada en la buena voluntad del docente, y no en un acto consciente derivado de los modelos educativos donde se contemple la definición de lo que se debe saber sobre las TIC. 


\section{Tabla 5. Semejanzas y diferencias en la Dimensión Social.}

\section{Semejanzas}

\section{Dimensión social}

\section{Infraestructura TIC de la universidad}

- Los docentes consideran que existe una brecha de acceso a las TIC, ya que, a pesar del esfuerzo de las universidades por adquirir dispositivos digitales y conectividad a internet, estos no siempre están disponibles en las aulas.

- También mencionan que las tecnologías están presentes en áreas de la universidad como los cubículos de los docentes y en la biblioteca, pero no en el aula, por ello no es pertinente incorporar estas herramientas en su práctica académica.

- UAEH: Los docentes afirman que se ha

fortalecido la infraestructura de la

universidad, sin embargo, señalan que otras universidades públicas y privadas tienen una estructura tecnológica superior.

- UCF: Los docentes consideran que la falta de infraestructura es un problema nacional, que repercute en el uso de las TIC en todas las universidades cubanas.

- UAEH: Los docentes consideran que los laboratorios de cómputo no son suficientes, aunque si cuentan con la tecnología necesaria para que se desarrollen practicas incorporando las TIC.

- UCF: Los docentes refieren que los laboratorios están en malas condiciones, con equipos lentos y descuidados, además de que son insuficientes, por lo que es difícil cambiar el paradigma tradicional de la impartición de las clases.

\section{Infraestructura TIC personal}

- UAEH: Los docentes mencionan que tienen dispositivos digitales personales y que no es difícil poder comprarlos, el acceso personal a internet es habitual.

- UCF: Los docentes mencionan que es muy difícil poder adquirir dispositivos digitales, el acceso personal a internet es en algunos casos imposible por su alto costo.

\section{Uso eficiente de las TIC en la docencia}

- Existe una percepción de que no se están aprovechando de forma eficiente las tecnologías disponibles en la universidad, principalmente porque hay una falta de conocimiento en cómo utilizarlas. utilizar las tecnologías, pero esta eficiencia está relacionada con tareas como enviar correo electrónico y desarrollar presentaciones electrónicas.

- Consideran que no poseen una alfabetización digital que les permita utilizar pedagógicamente las tecnologías disponibles, aun después de participar en cursos de formación para el uso de las TIC.

- El uso de software se relaciona principalmente con el diseño de presentaciones electrónicas y redacción de textos digitales.
- UAEH: En las aulas existen pizarrones electrónicos que no han sido usados de forma eficiente para cambiar las estrategias de enseñanza debido a la falta de capacitación para su uso, así como porque no se ha identificado la necesidad para usarlos. - UCF: en las aulas no hay presencia de dispositivos tecnológicos ni acceso a internet.

Fuente: Elaboración propia. 
Comparación de los cambios en la práctica docente por la incorporación de las TIC

El estudio demuestra que en la UCF existe una brecha de acceso que no permite generar actividades en el aula para usar las tecnologías como parte del proceso de enseñanza, en este sentido, la opinión de los docentes es que los dispositivos y la conectividad son insuficientes, en el caso de la UAEH aun cuando se manifiesta una suficiencia de infraestructura, el discurso afirma que las herramientas digitales han sido utilizadas de forma básica, como por ejemplo para enviar correos electrónicos o diseñar presentaciones digitales, incluso, se encuentran instalados en las aulas pizarrones electrónicos que no son utilizados, las semejanzas y diferencias identificadas se pueden leer en la Tabla 5.

Ante la percepción de la existencia de una brecha de infraestructura, los conocimientos de los docentes relativos a usar las TIC se vuelven escasos y dejan de ser importantes por lo que no son incorporados a la práctica docente, en este sentido, López y Ramírez
(2015), mencionan que poco sirve que desde hace 30 años se esté priorizando la dotación tecnológica en las universidades si éstas no están al alcance de los docentes, su servicio es deficiente, o bien se emplean en el marco de un modelo de aprendizaje tradicional, por lo que más que una oportunidad, la infraestructura tecnológica se convierte en un problema.

\section{CONCLUSIONES}

En esta dimensión, es necesario precisar que presuponer que la presencia de tecnologías en el aula conlleva un cambio en la práctica docente es una falacia, más aún cuando se cuentan con evidencias empíricas de que estas herramientas no ayudan a los docentes ni a los estudiantes en el aula en el sentido de cambiar la enseñanza (Gould, Cox y Brumby, 2016), ya que incluso su presencia genera distracciones auto-infringidas (Ravizza, Uitvlugt y Fenn, 2016) tales como revisar las redes sociales, leer noticias y otros contenidos no académicos, lo que representa una pérdida de tiempo en clase con un costo en el rendimiento escolar. 
En cuanto al alcance y eficiencia del uso de la infraestructura tecnológica la UCF se ubicó en un nivel de adopción mientras que la UAEH alcanzó un nivel de transformación, no obstante, los docentes de ambas universidades han calificado como insuficiente el acceso a dispositivos digitales e internet, lo que ha originado que no conciban a las tecnologías como herramientas disponibles para incorporarlas a sus actividades, aun cuando tienen la percepción de que su empleo se ha incrementado significativamente en sus espacios de trabajo.

También debe mencionarse que estudio demuestra que existe una subutilización de los dispositivos digitales debido a la insuficiente capacitación que se tiene para manipularlos, por otra parte, la alfabetización digital es una tarea pendiente en ambas universidades, ya que al parecer los programas institucionales de formación docente no traen como consecuencia el cambio de las estrategias de enseñanza.
Por otra parte la acumulación de la experiencia de la utilización de las TIC se encuentra limitada por el poco uso que se hace de la infraestructura a la que se tiene acceso originada por el escaso desarrollo de estrategias didácticas digitales, así, las insuficiencias de infraestructura se complementan con insuficiencias en el seguimiento al uso de las TIC que hacen los docentes en las aulas, esto pone en evidencia una discordancia entre el discurso político y normativo respecto a brindar una educación mediada por el uso de las tecnologías para construir nuevos escenarios pedagógicos y lo que realmente sucede en las aulas.

\section{BIBLIOGRAFÍA}

Alfaro, A., Fernández, M., y Alvarado, R. (2014). El uso de las TIC en la formación permanente del profesorado para la mejora de su práctica docente, Revista científica electrónica de educación y comunicación en la sociedad del conocimiento, 2(14). Recuperado de http://www.ugr.es/ sevimeco/revis taeticanet/numero141/Articulos/F ormato/190.pdf 
Bereday, G. (1968). El método comparativo en pedagogía. Barcelona: Editorial Herder.

Blanco, S. (2018). Marco común de competencia digital docente. Revista Iberoamericana de Educación a Distancia, 21(1), 369-370. Recuperado de http://educalab.es/documents/101 80/12809/Marco+competencia+di gital+docente+2017/afb079871ad6-4b2d-bdc8-58e9faeeccea

Bourdieu, P. (2012). La distinción. Criterio y bases sociales del gusto, Madrid, Editorial Taurus.

Bray, M. Adamson, B. y Mason, M. (2010). Educación comparada. Enfoques y Métodos. México: Granica.

Bray, M. y Thomas, R. (1995).

Levels of comparision in educational studies: Different Insights literatures and the value of multilevel analisys. Harvard Educational Review, 65(63), 472490. Recuperado de: http://hepgjournals.org/doi/abs/10. 17763/haer.65.3.g3228437224v4 877

Cabero, J. (2014). Formación del profesorado universitario en TIC. Aplicación del Método Delphi para la selección de los contenidos formativos. Educación $X X 1,17(1), 111-131$. Recuperado de http://www.redalyc.org/pdf/706/70 629509005.pdf
Cárdenas, M., Paret, D., Campos, R., Campos, G. (2017). Mediaciones en los usos sociales de Internet por estudiantes universitarios cubanos de Ciencias de la Información, Periodismo y Comunicación Social. Revista Cubana de Información en Ciencias de la Salud 28(2). Recuperado de http://www.rcics.sld.cu/index.php/ acimed/article/view/1070

Casillas, M., Ramírez, A., Carvajal, M., y Valencia, K. (2016). La integración de México a la sociedad de la información. En C. E. Téllez (Ed.), Derecho y TIC. Vertientes actuales. México: Infotec.

Cela, J., Esteve, V., Mon, González, F. y Gisbert, M. (2017). El docente en la sociedad digital: una propuesta basada en la pedagogía transformativa y en la tecnología avanzada.

Profesorado. Revista de Currículum y Formación de Profesorado, 21(1). Recuperado de

http://www.redalyc.org/html/567/5 6750681020/

Cook, T. y Rechardt, Ch. (1986). Métodos cualitativos $y$ cuantitativos en investigación evaluativa. Madrid: Ediciones Morata.

Creswell, J. y Plano, V. (2011). Designing and conducting mixed methods research. Thousand Oaks, CA: Sage. 
Denzin, N. y Lincoln, Y (2012). Manual de investigación cualitativa. Madrid: Gedisa.

Díaz, F. y Hernández, G. (2010). Estrategias docentes para un aprendizaje significativo. Una interpretación constructivista. México: Mc Graw Hill.

George, C. y Veytia, M. (2017). Nativos y migrantes digitales, riesgo de una conceptualización simplista, En Desafíos de la cultura digital para la educación. Chávez, B., González, R. y Lay, I. Jalisco: UDGvirtual. Recuperado de

http://biblioteca.udgvirtual.udg.mx /eureka/pudgvirtual/desafios_cult ura_digital.pdf

Glaser, B. y Strauss, A. (1967). The Discovery of Grounded Theory: strategies for qualitative research. Nueva York: Aldine de Gruyter.

Gould, S., Cox, A. y Brumby, D. (2016). Diminished control in crowdsourcing: An investigation of crowdworker multitasking behavior. ACM Transactions on Computer-Human Interaction, 23(3). Recuperado de http://discovery.ucl.ac.uk/1482232 /1/gould-et-al-crowdsourcedinterruption-tochi.final.pdf

Hilker, F. (1964). La pedagogie comparée. Introduction à son historie, sa théorie et sa practiqué. Paris: Institut Pédagogique National. http://www.unesco.org/new/es/un esco/themes/icts/lifelonglearning/higher-education/
Landman, T. (2011). Política comparada: una introducción a su objeto y métodos de investigación. Madrid: Alianza Editorial.

Lizarazo, D. y Andión, M. (2013). Símbolos digitales.

Representaciones de las TIC en la comunidad escolar. México: Siglo XXI Editores.

Martínez, L., Hinojo, F. y Rodríguez, A. (2017). Fortalezas, debilidades y concepciones que tienen los profesores al momento de implementar las TIC en sus procesos de enseñanza. Revista Científica Electrónica de Educación y Comunicación en la Sociedad del Conocimiento, 17(2), 297-316. Recuperado de http://eticanet.org/revista/index.ph p/eticanet/article/view/141

OEI (2018). Estudio sobre la inclusión de las TIC en los centros educativos de aulas fundación telefónica, Madrid: España. Recuperado de https://www.fundaciontelefonica.c om/arte_cultura/publicacioneslistado/pagina-itempublicaciones/itempubli/635/

Pérez, A. y Rodríguez, M. (2016). Evaluación de las competencias digitales autopercibidas del profesorado de educación primaria en Castilla y León (España). Revista de Investigación Educativa, 34(2), 399-415. Recuperado de https://revistas.um.es/rie/article/d ownload/215121/195051 
Ramírez, A. y Casillas, M. (2015). Los saberes digitales de los universitarios. En J. Micheli. Educación virtual y universidad, un modelo de evolución. pp. 77106. Serie Estudios Biblioteca de Ciencias Sociales y

Humanidades. México:

Universidad Autónoma

Metropolitana. Recuperado de https://www.uv.mx/personal/albra mirez/files/2015/06/saberes_digit ales_uam.pdf

Raventós, F. (1983). El fundamento de la metodología comparativa en educación. Edu car Pedagogía comparada. (3), 61-75.

Universidad Autónoma de Barcelona: Centro de Pedagogía Comparada. Recuperado de http://www.raco.cat/index.php/Edu car/article/view/42058/89969.

Ravizza, S., Uitvlugt, M. y Fenn, K. (2016). Logged In and Zoned Out: How Laptop Internet Use Relates to Classroom Learning. Psychological Science, Vol. 1, No. 10. Michigan State University, Sage: Michigan. Recuperado de http://journals.sagepub.com/doi/abs/10.1177/09567976166773 14 ?journalCode=pssa
Romero, M., Castejón, F., López, F. y Fraile A. (2017). Evaluación formativa, competencias comunicativas y TIC en la formación del profesorado. Comunicar: Revista Científica Iberoamericana de Comunicación y Educación, 25(52), pp. 73-82. Recuperado de https://www.revistacomunicar.com /verpdf. php? numero $=52 \&$ articulo $=$ 52-2017-07

Schriewer, J. (1993): El método comparativo y la necesidad de externalización: criterios metodológicos y conceptos sociológicos, en J. Schriewer y F. Pedró (Eds.), Manual de educación comparada (Vol. II) Teorías, investigaciones, perspectivas, pp. 189-251. Barcelona: Pomares-Corredor.

Stake, R. (1998). Investigación con estudio de casos. Madrid: Morata.

Strauss, A. y Corbin, J. (2002). Bases de la investigación cualitativa. Técnicas y procedimientos para desarrollar la teoría fundamentada. Universidad de Antioquía: Colombia.

Vargas, A. (1995). Estadística descriptiva e inferencial. Servicio de publicaciones de la Universidad de Castilla-La Mancha: Madrid. 